\title{
The value of diffusion tensor tractography delineating corticospinal tract in glioma in rat: validation via correlation histology
}

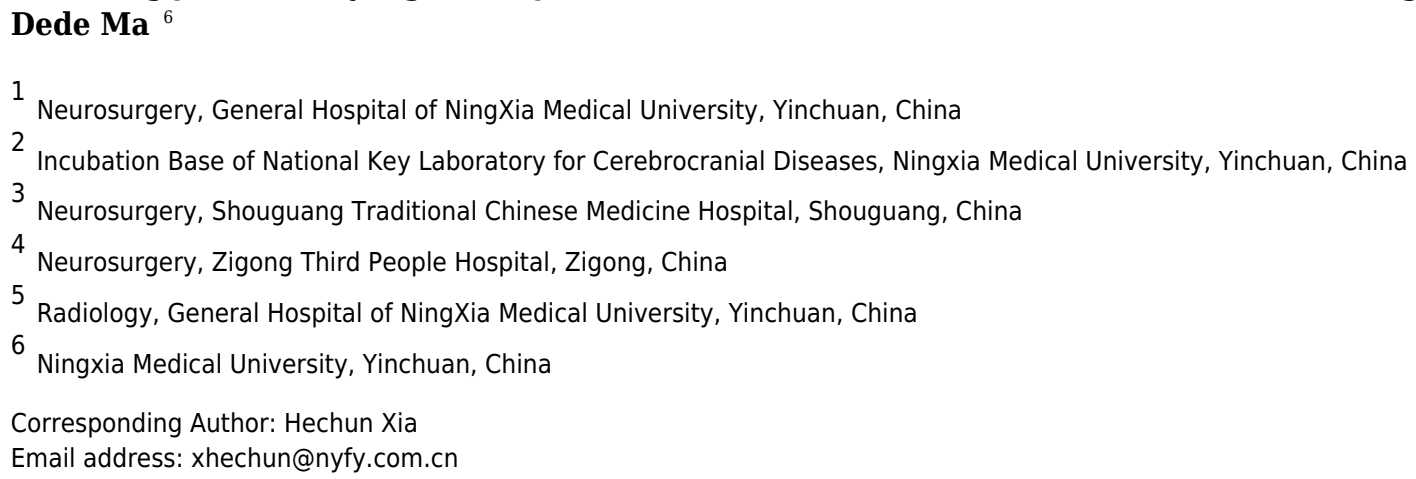

Background An assessment of the degree of white matter tract injury is important in neurosurgical planning for patients with gliomas. The main objective of this study was to assess the injury grade of the corticospinal tract (CST) in rats with glioma using diffusion tensor imaging (DTI). Methods Seventeen rats underwent 7.0T MRI on day 10 after tumor implantation. The apparent diffusion coefficient (ADC) and fractional anisotropy (FA) were acquired in the tumor, peritumoral and contralateral areas, and the ADC ratio (ipsilateral

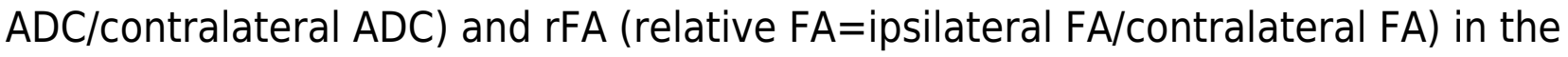
peritumoral areas were measured. The CST injury was divided into three grades and delineated by diffusion tensor tractography (DTT) reconstruction imaging. The fiber density index (FDi) of the ipsilateral and contralateral CST and rFDi (relative FDi=ipsilateral FDi/contralateral FDi) in the peritumoral areas were measured. After the mice were sacrificed, the invasion of glioma cells and fraction of proliferating cells were observed by hematoxylin-eosin ( $\mathrm{H}: \mathrm{E}$ ) and Ki67 staining in the tumor and peritumoral areas. The correlations among the pathology results, CST injury grade and DTI parameter values were calculated using a Spearman correlation analysis. One-way analysis of variance (ANOVA) was performed to compare the different CST injury grade by the rFA, rFDi and ADC ratio values. Results The tumor cells and proliferation index were positively correlated with the CST injury grade $(r=0.8857,0.9233, P<0.001)$. A negative correlation was demonstrated between the tumor cells and the rFA and rFDi values in the peritumoral areas ( $r=-0.8571$, 0.5588 ), and the proliferation index was negatively correlated with the rFA and rFDi values ( $r=-0.8571,-0.5588)$, while the ADC ratio was not correlated with the tumor cells or 
proliferation index. The rFA values between the CST injury grades ( 1 and 3,2 and 3) and the rFDi values in grades 1 and 3 significantly differed $(P<0.05)$. Conclusions DTI imaging may be used to quantify the injury degrees of CST involving brain glioma in rats. Our data suggest that these quantitative parameters may be used to enhance the efficiency of delineating the relationship between fiber tracts and malignant tumor. 
1 The value of diffusion tensor tractography delineating

2 corticospinal tract in glioma in rat: validation via

3 correlation histology

4

5 Xiaoxiong $\mathrm{Jia}^{1,2}$, Zhiyong $\mathrm{Su}^{3}$, Junlin $\mathrm{Hu}^{4}$, Hechun $\mathrm{Xia}^{1,2 \#}$, Hui Ma${ }^{1}$, Xiaodong Wang ${ }^{5}$, Jiangshu

$6 \mathrm{Yan}^{1}$, Dede $\mathrm{Ma}^{6}$

7

$8{ }^{1}$ Neurosurgery, General Hospital of NingXia Medical University, Yinchuan, China

$9{ }^{2}$ Incubation Base of National Key Laboratory for Cerebrocranial Diseases, Ningxia Medical

10 University, Yinchuan, China

$11{ }^{3}$ Neurosurgery, Shouguang Traditional Chinese Medicine Hospital, Shouguang, China

$12{ }^{4}$ Neurosurgery, Zigong Third People Hospital, Zigong, China

$13{ }^{5}$ Radiology, General Hospital of NingXia Medical University, Yinchuan, China

$14{ }^{6}$ NingXia Medical University, Yinchuan, China

Corresponding author:

Hechun Xia ${ }^{1,2 \#}$

Shengli Street 804, Yin Chuan, Ning Xia, 750000, China.

Email address: xhechun@nyfy.com.cn 
33 Abstract

34 Background

35 An assessment of the degree of white matter tract injury is important in neurosurgical 36 planning for patients with gliomas. The main objective of this study was to assess the 37 injury grade of the corticospinal tract (CST) in rats with glioma using diffusion tensor

\section{Methods}

Seventeen rats underwent 7.0T MRI on day 10 after tumor implantation. The apparent diffusion coefficient (ADC) and fractional anisotropy (FA) were acquired in the tumor, peritumoral and contralateral areas, and the $A D C$ ratio (ipsilateral $A D C /$ contralateral $A D C$ ) and $r F A$ (relative FA=ipsilateral FA/contralateral FA) in the peritumoral areas were measured. The CST injury was divided into three grades and delineated by diffusion tensor tractography (DTT) reconstruction imaging. The fiber density index (FDi) of the ipsilateral and contralateral CST and rFDi (relative FDi=ipsilateral FDi/contralateral FDi) in the peritumoral areas were measured. After the mice were sacrificed, the invasion of glioma cells and fraction of proliferating cells were observed by hematoxylin-eosin ( $\mathrm{H} \&$ E) and Ki67 staining in the tumor and peritumoral areas. The correlations among the pathology results, CST injury grade and DTI parameter values were calculated using a Spearman correlation analysis. One-way analysis of variance (ANOVA) was performed to compare the different CST injury grade by the rFA, rFDi and ADC ratio values.

\section{Results}

The tumor cells and proliferation index were positively correlated with the CST injury grade $(r=0.8857,0.9233, \mathrm{P}<0.001)$. A negative correlation was demonstrated between the tumor cells and the rFA and rFDi values in the peritumoral areas $(r=-0.8571,-0.5588)$, and the proliferation index was negatively correlated with the rFA and rFDi values ( $\mathrm{r}=-$ $0.8571,-0.5588)$, while the ADC ratio was not correlated with the tumor cells or proliferation index. The rFA values between the CST injury grades (1 and 3, 2 and 3) and the rFDi values in grades 1 and 3 significantly differed $(P<0.05)$.

\section{Conclusions}

DTI imaging may be used to quantify the injury degrees of CST involving brain glioma in rats. Our data suggest that these quantitative parameters may be used to enhance the efficiency of delineating the relationship between fiber tracts and malignant tumor.

\section{Introduction}

Gliomas are characterized by invasive growth along fiber tracts in the white matter 
68 (Chen et al. 2010). In particular, gliomas involving fiber tracts, such as CST, may result 69 in neurological deficits due to the disruption, displacement or deformation of the fibers (Laundre et al. 2005). Therefore, delineating the different injury degrees in the CST should provide important additional information for better neurological treatment planning.

DTI has been proven to classify damage to the fiber tracts and is used to quantitatively analyze the infiltration degree of the CST (Hervey-Jumper \& Berger 2014; Jeong et al. 2015). Diffusion tensor tractography (DTT) is a noninvasive tool that can be used to visualize major white matter tracts in 3 dimensions by setting the region of interest $(\mathrm{ROI})$ as the fiber path (Conti et al. 2014; Forster et al. 2015; Jeong et al. 2014). Recently, FDi values have been used to indicate the fiber density within the bundle passing through a unit volume, which can provide useful information for the assessment of the tumor border zone and peritumoral regions (Roberts et al. 2005; Stadlbauer et al. 2012). Some authors (Painter \& Hillen 2013; Witwer et al. 2002; Yu et al. 2005) have demonstrated that FA decreases in the white matter close to brain tumors, while ADC increases in the tumor core and peritumoral areas. Rosenstock et at. showed that the FA value were lower and the ADC value were higher in peritumoral areas, which indicated that the FA and ADC value were affected at the peritumoral level (Rosenstock et al. 2017). Additionally, tumor cell proliferation and invasion could result in a disruption of CST, and the degree of injury may reflect its biological behavior (Gao et al. 2017; Painter \& Hillen 2013).

Previous studies have shown that DTI imaging can be used to assess the degree of damage in fiber tracts, and DTI has been used in neurological examinations of patients. However, to the best of our knowledge, whether the rFA, rFDi and ADC ratio, which are hypothesized as quantitative parameters in DTI imaging, can be used to assess the injury grade in the CST or are correlated with pathology results in rats with glioma has not been established.

Therefore, in this study, the rFA, rFDi and ADC ratio were measured and evaluated in regions of interest (ROIs) in the white matter in peritumoral areas. Furthermore, different degrees of CST injury were reconstructed and delineated by DTT. Importantly, in rats with glioma, the correlation between the gliomas and the damage to the CST was addressed by describing the DTI parameters in terms of the invasion and proliferation of the tumor cells in the peritumoral areas.

\section{Materials and methods}

Rats and glioma cell injection

The animal studies were performed using protocols approved by the General Hospital of Ningxia Medical University Animal Care Committee (\#2013-81260373). Seventeen adult 
103 SD rats (200-250 $\mathrm{g}$ in body weight) were used in this study. Rat glioma cells (C6) were 104 cultured in Dulbecco's modified Eagle's medium supplemented with $10 \%$ fetal bovine 105 serum. The procedures used to engraft the $\mathrm{C} 6$ tumors in the rat brains have been reported 106 in detail in previous studies involving rat glioma models (Asanuma et al. 2008; Van Den 107 Berge et al. 2015; Zhanfeng et al. 2015). In total, 17 glioma-bearing rats were used. For 108 the implantations, each animal was anesthetized using $0.5 \%$ sodium pentobarbital ( 1 $\mathrm{ml} / .1 \mathrm{~kg}$, intraperitoneal), and a suspension of C6 cells $(1 \mu \mathrm{L} / \mathrm{min}, 10 \mu \mathrm{L})$ was stereotactically injected into the cortex at a depth of $5 \mathrm{~mm}$. The surgeries were performed under sterile conditions.

112 MRI protocol

113 All imaging was performed at 7.0T using a horizontal bore BRUKER Biospec 70/20 114 Advance MRI instrument (Bruker, Karlsruhe, Germany) at the National Center for 115 Nanoscience and Technology in Beijing, China. The radiofrequency (RF) coil used was a 116 surface coil. The rats were anesthetized using $0.5 \%$ sodium pentobarbital $(1 \mathrm{ml} / .1 \mathrm{~kg}$, 117 intraperitoneal) and then placed into a head holder with a bite-bar and ear bars to 118 immobilize the head. The body temperature was maintained at $37.5^{\circ} \mathrm{C}$ using a hot water119 circulating bath. The MRI scans were performed 10 days after the cell implantation.

120 The following T2-weighted imaging parameters were used to obtain the images: repetition 121 time $(T R)=2000 \mathrm{~ms}$, echo time $(T E)=20 \mathrm{~ms}$, field of vision $(F O V)=40 \times 40 \mathrm{~mm}^{2}$, slice 122 thickness $=1.0 \mathrm{~mm}$, acquisition matrix $=256 \times 256, \mathrm{NEX}=2$, flip angle $=90^{\circ}$, and number of 123 slices=20. The following DTI imaging parameters were used to obtain the images: $\mathrm{TR}=5000 \mathrm{~ms}$, TE=27 $\mathrm{ms}, \mathrm{FOV}=40 \mathrm{~mm} \times 40 \mathrm{~mm}^{2}$, slice thickness=1.0 mm, acquisition matrix $=128 \times 128$, flip angle $=90^{\circ}$, number of slices $=20$, and number of gradients $=25$. After the image acquisition, the data were transferred to a MATLAB workstation for analysis. $\mathrm{ROI}$ measurements

128 The fractional anisotropy (FA), apparent diffusion coefficient (ADC) and fiber density index (FDi) values were measured from regions of interest (ROls) chosen by two experienced neuroradiologists (Dr. Wang and Dr. Huang) with at least seven years of work experience (Price et al. 2003). The FA, ADC and FDi values were calculated in the following ROls, which were both contralateral and ipsilateral to the tumor: in the tumor and peritumoral areas. To enhance the accuracy of the measurement, the ROls were selected based on five different brain spots in each area (Blasiak et al. 2010; Lu et al. 2003). The FA, ADC and FDi value measurements in the contralateral and ipsilateral peritumoral areas were used to calculate the $A D C$ ratio (ipsilateral ADC/contralateral ADC), rFA (relative FA=ipsilateral FA/contralateral FA) and rFDi (relative FDi=ipsilateral 
$138 \mathrm{FDi} /$ contralateral FDi).

139 CST injury grade

140 The CST originates in the motor cortex area and then travels through the posterior limb 141 of the internal capsule to enter the cerebral peduncle. Therefore, the ROls of the CST 142 were usually placed in the subcortical white matter of the precentral gyrus (Brodmann 4) 143 and premotor cortex (Brodmann 6) area (ROI), the posterior limb of the internal capsule 144 (ROI), or the entire cerebral peduncle (ROI). To isolate fibers of the CST with the "AND" operation of DTT software, the traces that passed through these ROIs (two or three ROIs) were considered to be components of the CST (Holodny et al. 2005; Suzuki et al. 2009). During fiber tracking by diffusion tensor tractography, the DTI data were processed and analyzed off-line using the TrackVis software package and a FA value of 0.20 . In our study, the motor cortex area (initial ROI) and cerebral peduncle (target ROI) were chosen by two experienced neuroradiologists (XDW, XYH).

In this study, the white matter tracts in seventeen glioma-bearing rats were classified according to the criteria of displacement, infiltration and disruption (Gao et al. 2017; Witwer et al. 2002) as follows: 1) grade 1: displaced and edematous if the tracts displayed a normal anisotropy signal relative to the corresponding tracts in the contralateral hemisphere, displacement of the fibers by the tumor and some peritumoral edema delineated by the DTT imaging (Fig. S1). 2) grade 2: infiltrated if the tracts showed decreased anisotropy but remained intact without significant disruption; 3) grade 3 : disrupted if anisotropy was markedly reduced such that the tract could not be identified on the FA map, and interruption of the white matter tracts can be observed on DTT imaging.

\section{Animal test}

The animal test was performed one day before the MRI scan by two observers ( $\mathrm{Dr}$. $\mathrm{H}$ and Dr. S) in a blinded fashion. Two tests (modified from Garcia) were used to evaluate the motor function of the rats (Garcia et al. 1995), and the scores ranged from 1 (minimum) to 6 (maximum). The grading scale for motor deficit (modified from Bederson) was divided into two grades (moderate: scores 3-6, and severe: scores 1-2) as described below (Bederson et al. 1986).

Spontaneous activity: The rat was observed for two minutes in a transparent plastic cube $30 \mathrm{~cm}$ in length. Climbing test: The animal was placed and climbed the wire mesh wall. The rat's tail was held, and the rat was pulled off, during which the strength of the rat's four limbs was noted.

Histology and immunohistochemistry

After completing the MRI experiments, the rats were sacrificed 10 days after implantation, 
174 and the whole brains of the rats were removed from the skull and stained using 175 hematoxylin-eosin (H\&E) and Ki67 (Del Duca et al. 2004). The fraction of glioma invasion 176 cells among the total number of cells in the peritumoral areas was assessed in $\mathrm{H} \& \mathrm{E}$ 177 stained sections in a high-powered field (HPF), and the regions of measurement were 178 selected based on five different brain spots in each area. Using Ki67 staining, we 179 evaluated the fraction of proliferating cells in each peritumoral regions and selected five 180 different brain spots (Smith et al. 2017). The histological specimens were analyzed to determine the regions that corresponded to the quantitative MRI measurements (Wang \& Zhou 2012). These tumor cells were compared with the data obtained using the DTI imaging. The maps used to obtain the microscopic cell counts were derived using the Image-Pro Plus software package.

\section{Statistical analysis}

The statistical calculations were performed using the GraphPad Prism 5 software package and the statistical package SPSS (Version 21, SPSS). The FA, ADC and FDi data are presented as the mean \pm standard deviation (SD) as indicated. A paired-sample $t$-test was applied to analyze the significance of the differences observed in the parameters between the tumoral and peritumoral areas, between the contralateral normal area and tumoral areas, and between the contralateral normal area and peritumoral areas. A P-value less than 0.05 was considered to indicate a significant difference. The correlations between the CST injury grades and the rFA and rFDi in the tumor cells were calculated using a Spearman correlation analysis. The rFA and rFDi values were compared among groups with different CST injury degrees with a one-way analysis of variance (ANOVA).

\section{Results}

\section{Summary of quantitative parameters and CST injury grades in 17 rats}

The mean \pm standard deviation of the FA, ADC and FDi were measured in the tumoral and peritumoral areas and contralateral hemisphere tissue (Table S1, S3 and Fig. 1A,B,C). The DTI analysis and histology results of the peritumoral areas are shown in Table S2. The tumor cells and proliferation index were positively correlated with the CST injury grade $(\mathrm{r}=0.8857,0.9233, \mathrm{P}<0.001)$. A negative correlation was observed between the tumor cells and the rFA and rFDi values in the peritumoral areas $(r=-0.8571,-0.5588$, respectively), and the proliferation index was negatively correlated with the rFA and rFDi values ( $r=-0.8698,-0.5856$, respectively), while the ADC ratio was not correlated with the tumor cells or proliferation index (Fig. 1D, E).

\section{Diffusion tensor imaging analysis and CST injury grades}


209 The rFA values significantly differed between the CST injury grades ( 1 and 3, 2 and 3)

$210 \quad(P<0.05)$ (Fig. 2A); the rFDi values significantly differed between the grades (1 and 3 )

$211(\mathrm{P}<0.05)$ (Fig. 2B), while the ADC ratio (Fig. 2C) and $r F A$ and $r F D i$ values in the remaining

212 CST injury grade groups did not significantly differ.

213 Histology and immunohistochemistry of peritumoral areas

214 The tumor cells in the peritumoral areas were assessed in H\&E stained sections and

215 compared among the different CST injury grade groups of seventeen rats. Grade 1 (Fig.

216 3A) had a median value of $39 \%$ per high powered field under 40x magnification; grade 2

217 (Fig. 3B) had a median of $48 \%$ per HPF, and grade 3 (Fig. 3C) had a median of $57 \%$ per

218 HPF. The tumor cells significantly differed between the CST injury grade groups (1 and

219 2, 1 and 3, 2 and 3) (Fig. 3D).

220 The proliferation index in the peritumoral areas was assessed and compared among the 221 different CST injury grade groups by using Ki67 immunohistochemistry staining. In grade

2221 (Fig. 3E), the fraction of Ki67 positive cells was 16\% under 40x magnification; in grade 2232 (Fig. 3F), the median was $24 \%$, and in grade 3 (Fig. 3G), the median was $44 \%$. The 224 proliferation index significantly differed among the grades ( 1 and 3 and 2 and 3$)(P<0.05)$, while in the remaining groups (1 and 2), no significant differences were observed (Fig. $3 \mathrm{H})$.

\section{Displacement of corticospinal fibers}

The T2WI and DTI scans were performed in seventeen glioma-bearing rats. On the conventional $\mathrm{T} 2 \mathrm{WI}$, the tumors showed irregular mixed signals in regions involved with the right motor cortex (Fig. 4A). On the FA maps, normal diffusion anisotropy signals were observed in the right hemisphere relative to those in the contralateral areas (Fig. 4B,C). In the DTT, the initial point was the motor cortex area, and the cerebral peduncle was the seed point. Furthermore, the 3D reconstruction map showed that the right CST was displaced anterolaterally by the mass lesion compared with the left fibers (Fig. 4D).

\section{Disruption of corticospinal fibers}

As shown in Fig. 5, the corresponding fiber tracts were disrupted by the tumors using 3D reconstruction DTT imaging. The conventional MRI scans revealed a large hypo-intense lesion on T2W imaging involving the right motor area and subcortical regions (Fig. 5A). On the FA color maps, the anisotropy in the ipsilateral hemisphere was markedly reduced compared with that in the left hemisphere such that the fibers could not be tracked (Fig. 5B). Fig. 5C shows the DTT reconstructed complex fibers without the target and initial regions of interest (ROIs) in the superior view. Compared with the contralateral hemisphere, the fibers on the right side were disrupted and affected by the tumor (light 
244 yellow areas). Green, anterior/posterior; blue, inferior/superior; and red, right/left. The 245 reconstruction of the DTT images showed that the right CST was no longer present 246 compared with the contralateral fibers in the left hemisphere (Fig. 5D).

\section{Discussion}

248 Resection of gliomas involving motor functional areas requires a detailed understanding 249 of the relationships between the motor cortex and surrounding brain tissue and 250 subcortical white matter fibers (Gao et al. 2017; Hervey-Jumper \& Berger 2014). The aim 251 of this study was to quantitatively analyze the degree of damage to the fibers affected by 252 gliomas and evaluate the role of DTI imaging in visualizing the CST in the vicinity of the 253 motor cortex.

254 DTI parameters are used to evaluate different grades of gliomas, assess the extent of peritumoral edema and reflect the degree of malignant tumor infiltration (Budde et al. 2011; Harsan et al. 2010; Hou et al. 2018). Our statistical results showed that the differences in the FA, ADC, and FDi between the tumor areas and peritumoral areas are significant and that the rFA and rFDi values were negatively correlated with the tumor cells and proliferation index in the peritumoral areas, while the ADC ratio values did not significant differ. Using H\&E and Ki67 staining, in this study, the higher number of tumor cells and proliferative index in the peritumoral areas indicate high invasion into the white matter. In our experience, the FA and FDi values decrease in peritumoral areas likely due to tumor infiltration and proliferation. Consequently, invasion of tumor cells could lead to damage to fibers in gliomas. DTI imaging can indicate whether anatomically intact fibers are present in abnormally appearing areas of the brain and characterize the integrity of white matter tracts in patients with brain tumors (Stadlbauer et al. 2012; Witwer et al. 2002). In this study, we demonstrated that the rFA and rFDi values significantly differed based on the grade of CST damage. Some studies have found that the FA value is related to the integrity of subcortical fiber tracts (Harsan et al. 2010). Chen $\mathrm{Y}$ et al. found that the FDi could be used to evaluate fiber disruption and tumor infiltration (Chen et al. 2010). White matter tracts were classified and predictive of total resection or partial resection (Jia et al. 2013). The degree of CST injury from glioma was correlated with the rFA and rFDi values, suggesting that DTI can reflect different degrees of injury from glioma in CST area and predict more useful information regarding the relationship between the nerve fibers and the lesions. Therefore, DTI parameters could be used to evaluate peritumoral areas along the path of CST fibers that have been affected by tumors. The goal of charactering the CST injury grades and delineating the different components 
279 of the fiber tracts is to provide more accurate information to describe the spatial 280 relationship between a malignant tumor and normal tissues. In our study, we showed that 281 the tracking of the CST could be performed, visualized and distinguished by DTT 282 reconstruction imaging. DTT can be used to visualize major white matter tracts by setting 283 the ROI on the path of the fibers using anatomic structures (Baldoli et al. 2015; Kleiser et 284 al. 2010). In our study, we verified the efficacy of a protocol that uses initial and target ROIs to delineate CST involved in diffusely infiltrating gliomas in rats. Better delineation of the CST injury grade could provide important additional information for tumor removal assessment.

Although we evaluated the fiber tracts by DTI parameters compared with histology, we suggest performing experiments to delineate white matter using additional staining. The further development of our findings could expand the range of these applications beyond the limit of the motor areas and increase the number of animal models.

\section{Conclusions}

In conclusion, our results demonstrate that DTI imaging may quantitatively evaluate the injury degrees in fiber tracts involving brain gliomas in rats. Our data suggest that these quantitative parameters may be used to enhance the efficiency of delineating the relationship between fiber tracts and tumors.

\section{Acknowledgments}

The authors are grateful to Enshan Han and Xiaoli Liu (Neuropathologist Department, General Hospital of NingXia Medical University, Yinchuan, Ningxia, China) for performing the histological examination.

6




\section{References}

Asanuma T, Doblas S, Tesiram YA, Saunders D, Cranford R, Pearson J, Abbott A, Smith N, and Towner RA. 2008. Diffusion tensor imaging and fiber tractography of C6 rat glioma. J Magn Reson Imaging 28:566-573. 10.1002/jmri.21473

Baldoli C, Scola E, Della Rosa PA, Pontesilli S, Longaretti R, Poloniato A, Scotti R, Blasi V, Cirillo S, Iadanza A, Rovelli R, Barera G, and Scifo P. 2015. Maturation of preterm newborn brains: a fMRI-DTI study of auditory processing of linguistic stimuli and white matter development. Brain Struct Funct 220:3733-3751. 10.1007/s00429-014-0887-5

Bederson JB, Pitts LH, Tsuji M, Nishimura MC, Davis RL, and Bartkowski H. 1986. Rat middle cerebral artery occlusion: evaluation of the model and development of a neurologic examination. Stroke 17:472-476.

Blasiak B, Tomanek B, Abulrob A, Iqbal U, Stanimirovic D, Albaghdadi H, Foniok T, Lun X, Forsyth P, and Sutherland GR. 2010. Detection of T(2) changes in an early mouse brain tumor. Magn Reson Imaging 28:784-789. 10.1016/j.mri.2010.03.004

Budde MD, Janes L, Gold E, Turtzo LC, and Frank JA. 2011. The contribution of 
gliosis to diffusion tensor anisotropy and tractography following traumatic brain injury: validation in the rat using Fourier analysis of stained tissue sections. Brain 134:2248-2260. 10.1093/brain/awr161

340

Chen Y, Shi Y, and Song Z. 2010. Differences in the architecture of low-grade and high-grade gliomas evaluated using fiber density index and fractional anisotropy. J Clin Neurosci 17:824-829. 10.1016/j.jocn.2009.11.022

Conti A, Raffa G, Granata F, Rizzo V, Germano A, and Tomasello F. 2014. Navigated transcranial magnetic stimulation for "somatotopic" tractography of the corticospinal tract. Neurosurgery 10 Suppl 4:542-554; discussion 554. $10.1227 /$ neu.0000000000000502

Del Duca D, Werbowetski T, and Del Maestro RF. 2004. Spheroid preparation from hanging drops: characterization of a model of brain tumor invasion. $J$ Neurooncol 67:295-303.

Forster MT, Hoecker AC, Kang JS, Quick J, Seifert V, Hattingen E, Hilker R, and Weise LM. 2015. Does navigated transcranial stimulation increase the accuracy of tractography? A prospective clinical trial based on intraoperative motor evoked potential monitoring during deep brain stimulation. Neurosurgery 76:766-775; discussion $775-766$. 10.1227/neu.0000000000000715

Gao B, Shen X, Shiroishi MS, Pang M, Li Z, Yu B, and Shen G. 2017. A pilot study 
of pre-operative motor dysfunction from gliomas in the region of corticospinal tract: Evaluation with diffusion tensor imaging. PLoS One 12:e0182795. 10.1371/journal.pone.0182795

Garcia JH, Wagner S, Liu KF, and Hu XJ. 1995. Neurological deficit and extent of neuronal necrosis attributable to middle cerebral artery occlusion in rats. Statistical validation. Stroke 26:627-634; discussion 635.

Harsan LA, Paul D, Schnell S, Kreher BW, Hennig J, Staiger JF, and von Elverfeldt D. 2010. In vivo diffusion tensor magnetic resonance imaging and fiber tracking of the mouse brain. NMR Biomed 23:884-896. 10.1002/nbm.1496

Hervey-Jumper SL, and Berger MS. 2014. Role of surgical resection in low- and high-grade gliomas. Curr Treat Options Neurol 16:284. 10.1007/s11940-0140284-7

Holodny AI, Watts R, Korneinko VN, Pronin IN, Zhukovskiy ME, Gor DM, and Ulug A. 2005. Diffusion tensor tractography of the motor white matter tracts in man: Current controversies and future directions. Ann N Y Acad Sci 1064:88-97. 10.1196/annals.1340.016

Hou Z, Cai X, Li H, Zeng C, Wang J, Gao Z, Zhang M, Dou W, Zhang N, Zhang L, and Xie J. 2018. Quantitative Assessment of Invasion of High-Grade Gliomas Using Diffusion Tensor Magnetic Resonance Imaging. World Neurosurg 113:e561-e567. 10.1016/j.wneu.2018.02.095 
377 Jeong JW, Asano E, Juhasz C, and Chugani HT. 2014. Quantification of primary

378

379

380

381

382

383

384

385

386

387

388

389

390

391

392

393

394

395

396 motor pathways using diffusion MRI tractography and its application to predict postoperative motor deficits in children with focal epilepsy. Hum Brain Mapp 35:3216-3226. 10.1002/hbm.22396

Jeong JW, Lee J, Kamson DO, Chugani HT, and Juhasz C. 2015. Detection of hand and leg motor tract injury using novel diffusion tensor MRI tractography in children with central motor dysfunction. Magn Reson Imaging 33:895-902. 10.1016/j.mri.2015.05.003

Jia XX, Yu Y, Wang XD, Ma H, Zhang QH, Huang XY, and Xia HC. 2013. fMRIdriven DTT assessment of corticospinal tracts prior to cortex resection. Can J Neurol Sci 40:558-563.

Kleiser R, Staempfli P, Valavanis A, Boesiger P, and Kollias S. 2010. Impact of fMRI-guided advanced DTI fiber tracking techniques on their clinical applications in patients with brain tumors. Neuroradiology 52:37-46. $10.1007 / \mathrm{s} 00234-009-0539-2$

Laundre BJ, Jellison BJ, Badie B, Alexander AL, and Field AS. 2005. Diffusion tensor imaging of the corticospinal tract before and after mass resection as correlated with clinical motor findings: preliminary data. $A J N R A m J$ Neuroradiol 26:791-796.

Lu S, Ahn D, Johnson G, and Cha S. 2003. Peritumoral diffusion tensor imaging of 
high-grade gliomas and metastatic brain tumors. AJNR Am J Neuroradiol 24:937-941.

Painter KJ, and Hillen T. 2013. Mathematical modelling of glioma growth: the use of Diffusion Tensor Imaging (DTI) data to predict the anisotropic pathways of cancer invasion. $J$ Theor Biol 323:25-39. 10.1016/j.jtbi.2013.01.014

Price SJ, Burnet NG, Donovan T, Green HA, Pena A, Antoun NM, Pickard JD, Carpenter TA, and Gillard JH. 2003. Diffusion tensor imaging of brain tumours at 3T: a potential tool for assessing white matter tract invasion? Clin Radiol 58:455-462.

Roberts TP, Liu F, Kassner A, Mori S, and Guha A. 2005. Fiber density index correlates with reduced fractional anisotropy in white matter of patients with glioblastoma. AJNR Am J Neuroradiol 26:2183-2186.

Rosenstock T, Giampiccolo D, Schneider H, Runge SJ, Bahrend I, Vajkoczy P, and Picht T. 2017. Specific DTI seeding and diffusivity-analysis improve the quality and prognostic value of TMS-based deterministic DTI of the pyramidal tract. Neuroimage Clin 16:276-285. 10.1016/j.nicl.2017.08.010

Smith SJ, Diksin M, Chhaya S, Sairam S, Estevez-Cebrero MA, and Rahman R. 2017. The Invasive Region of Glioblastoma Defined by 5ALA Guided Surgery Has an Altered Cancer Stem Cell Marker Profile Compared to Central Tumour. Int J Mol Sci 18. 10.3390/ijms18112452 
417 Stadlbauer A, Hammen T, Buchfelder M, Bachmair J, Dorfler A, Nimsky C, and 418 Ganslandt O. 2012. Differences in metabolism of fiber tract alterations in gliomas: a combined fiber density mapping and magnetic resonance spectroscopic imaging study. Neurosurgery 71:454-463. 10.1227/NEU.0b013e318258e332

Suzuki Y, Yagi K, Kodama T, and Shinoura N. 2009. Corticospinal tract extraction combining diffusion tensor tractography with FMRI in patients with brain diseases. Magn Reson Med Sci 8:9-16.

Van Den Berge N, Vanhove C, Descamps B, Dauwe I, van Mierlo P, Vonck K, Keereman V, Raedt R, Boon P, and Van Holen R. 2015. Functional MRI during Hippocampal Deep Brain Stimulation in the Healthy Rat Brain. PLoS One 10:e0133245. 10.1371/journal.pone.0133245

Wang S, and Zhou J. 2012. Diffusion tensor magnetic resonance imaging of rat glioma models: a correlation study of MR imaging and histology. J Comput Assist Tomogr 36:739-744. 10.1097/RCT.0b013e3182685436

Witwer BP, Moftakhar R, Hasan KM, Deshmukh P, Haughton V, Field A, Arfanakis K, Noyes J, Moritz CH, Meyerand ME, Rowley HA, Alexander AL, and Badie B. 2002. Diffusion-tensor imaging of white matter tracts in patients with cerebral neoplasm. $J \quad$ Neurosurg 97:568-575. 10.3171/jns.2002.97.3.0568 
437 Yu CS, Li KC, Xuan Y, Ji XM, and Qin W. 2005. Diffusion tensor tractography in 438 patients with cerebral tumors: a helpful technique for neurosurgical planning 439 and postoperative assessment. Eur $J$ Radiol 56:197-204. 10.1016/j.ejrad.2005.04.010

441

442 


\section{Figure 1}

Comparisons of the FA, ADC, and FDi values

Comparisons of the FA (A), ADC (B), and FDi (C) values in different brain regions and correlation between the DTI quantitative parameters (rFA, rFDi and ADC ratio) and histological results of peritumoral areas. (D), (E), A negative correlation is observed among the tumor cells, proliferation index and rFA and rFDi values in the peritumoral areas, while the ADC ratio was not correlated with the tumor cells and proliferation index. Tumor cells $(\%)=$ density of glioma invasion cells present in the peritumoral areas per high powered field; Proliferation index $(\%)=$ the fraction of proliferating cells in each peritumoral region;TA=tumoral areas; $\mathrm{PA}=$ peritumoral areas; $\mathrm{Con}=$ contralateral. Values are mean $\pm \mathrm{SD}$. $\mathrm{n}=17 . * \mathrm{P}<0.05, * * \mathrm{P}<0.01$ and $* * * \mathrm{P}<0.001$.
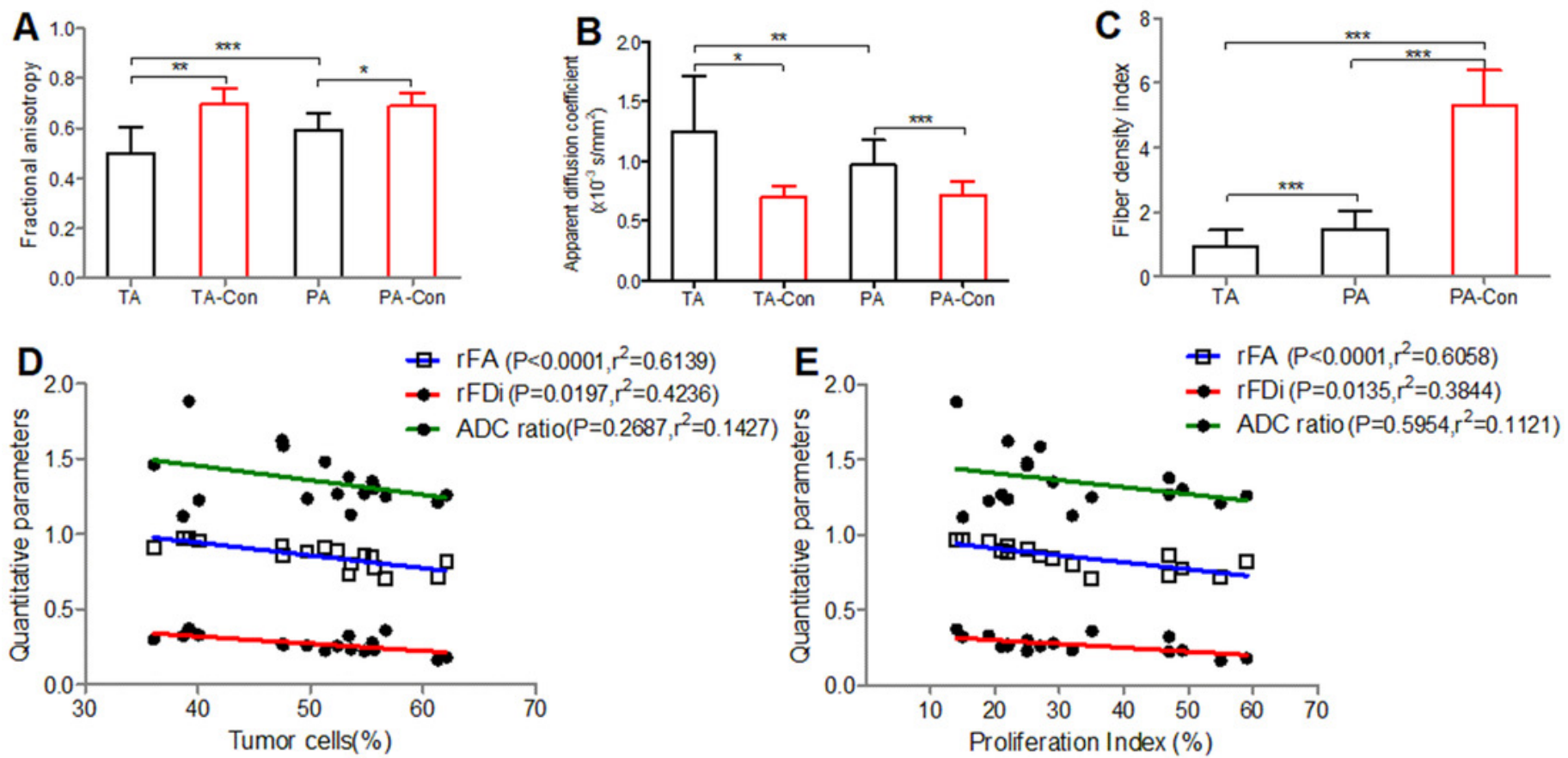


\section{Figure 2}

Comparison of the rFA, rFDi, and ADC ratio.

Comparison of the rFA (A), rFDi (B), and ADC ratio (C) among different CST injury grades in peritumoral areas. Grade1(black), Grade2(red), Grade3(blue); rFA=ipsilateral FA/contralateral FA; rFDi=ipsilateral FDi/contralateral FDi; $A D C$ ratio=ipsilateral $A D C /$ contralateral $A D C ; n=17$. $* P<0.05, * * P<0.01$ and $* * * P<0.001$.
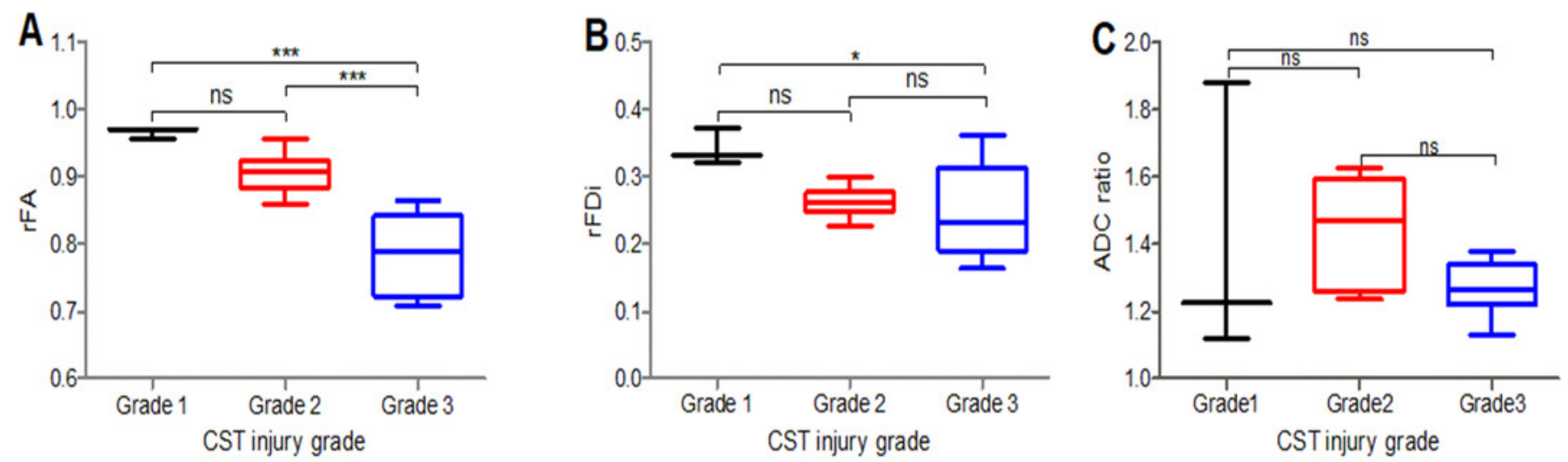


\section{Figure 3}

Three CST injury grades show different infiltration degrees and growth rates in peritumoral areas.

(A,B,C) H\&E sections were used to assess the percentage of tumor cells among the total number of cells per high powered field under 40x magnification. (A) Grade 1 samples had a median tumor cell fraction of 39\%. (B) Grade 2 samples had a median tumor cell fraction of $48 \%$. (C) Grade 3 samples had a median tumor cell fraction of 57\%. (D) Comparison of tumor cells among different CST injury grades in peritumoral areas. (E,F,G) Ki67 immunohistochemistry staining under 40x magnification to record the positive fraction in peritumoral areas. (E) Grade 1 samples had a median Ki67 positive cell fraction of 16\%. (F) Grade 2 samples had a median Ki67 positive cell fraction of 24\%. (G) Grade 3 samples had a median Ki67 positive cell fraction of 44\%. (H) Comparison of the proliferation index among different CST injury grades in peritumoral areas. (D,H) Grade1(black), Grade2(red), Grade3(blue); $\mathrm{n}=17 . * P<0.05, * * P<0.01$ and $* * * P<0.001$. 

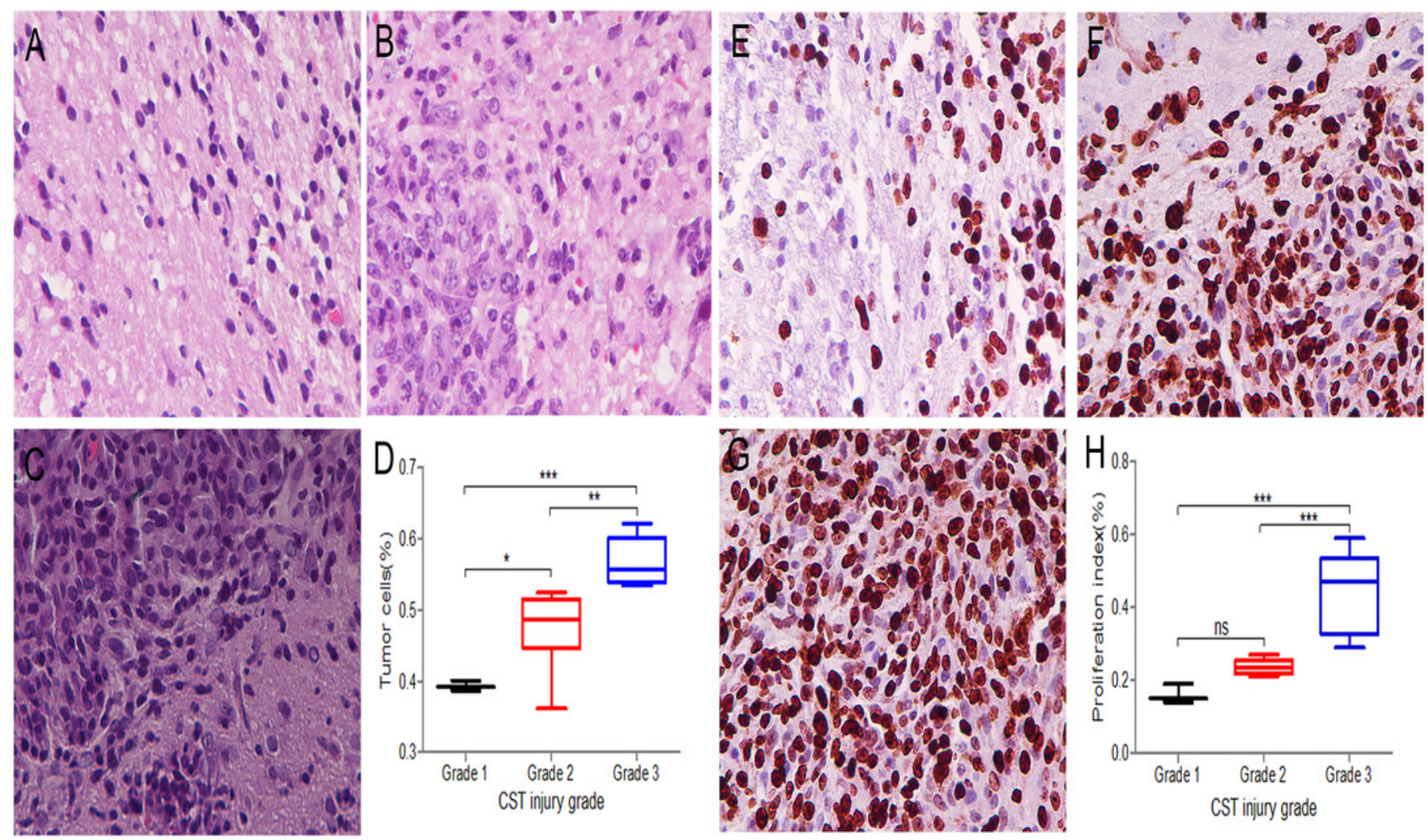


\section{Figure 4}

Displacement of corticospinal tract (CST) with CST injury grade 2 .

(A) T2W image showing a tumor with a mixed signal in the vicinity of the right motor cortex.

(B,C) Representative FA color imaging and FA imaging. The anisotropy signal decreased compared with that in the contralateral hemisphere. Green, anterior/posterior; blue, inferior/superior; and red, right/left. (D) Representative 3D reconstruction DTT imaging; the right CST is displaced anterolaterally by the mass lesion (light blue irregular solid shape) compared with the left fibers; right motor cortex (red solid sphere), left normal anatomical motor cortex (dark blue sphere), right cerebral peduncle (yellow sphere), left cerebral peduncle (green sphere); $\mathrm{R}=$ right side. 

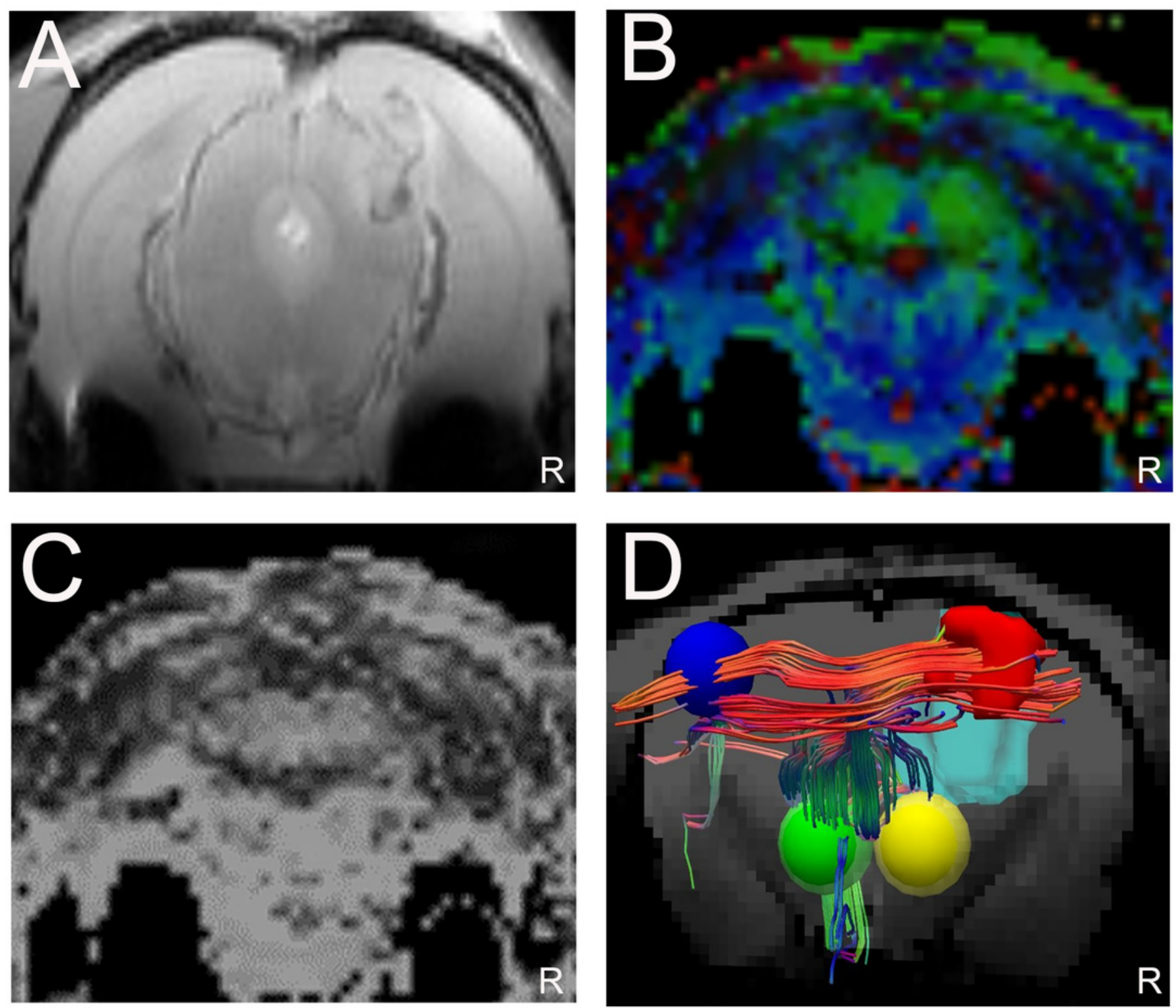


\section{Figure 5}

Disruption of corticospinal tract (CST) with CST injury grade 3.

(A) T2W image showing a large tumor with necrosis in the right hemisphere. (B)

Representative FA color imaging. Anisotropy was markedly reduced. (C) Representative DTT reconstruction complex fibers without the target and initial regions of interest (ROIs) in the superior view. Green, anterior/posterior; blue, inferior/superior; and red, right/left. (D) Representative 3D DTT imaging; the corresponding CST fibers are no longer present in their normal anatomical location within the right motor area involving the tumor (light yellow areas); right motor cortex (red irregular area), left motor cortex (light blue area), right cerebral peduncle (yellow sphere), left cerebral peduncle (orange sphere). $\mathrm{R}=$ right side. 

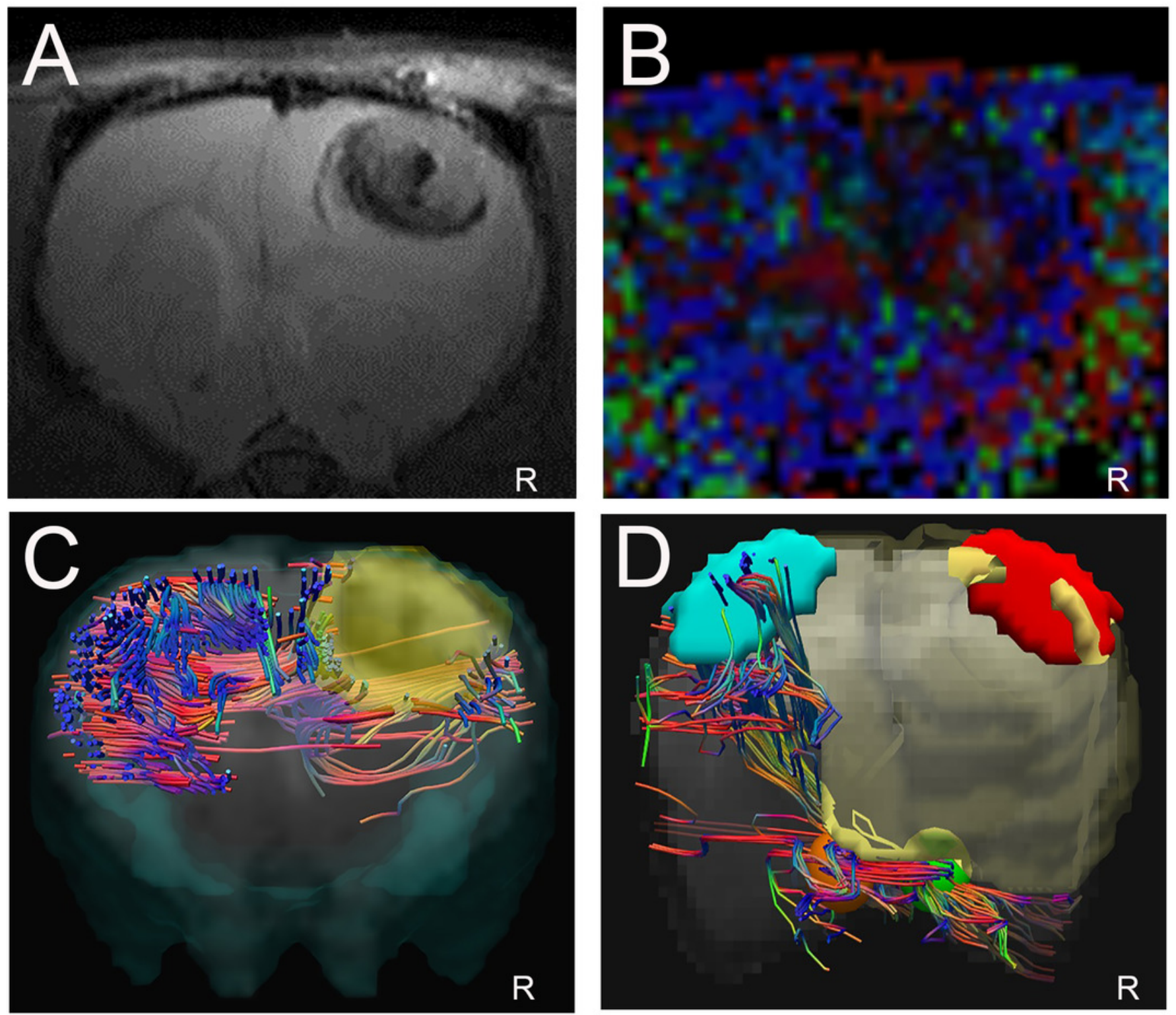